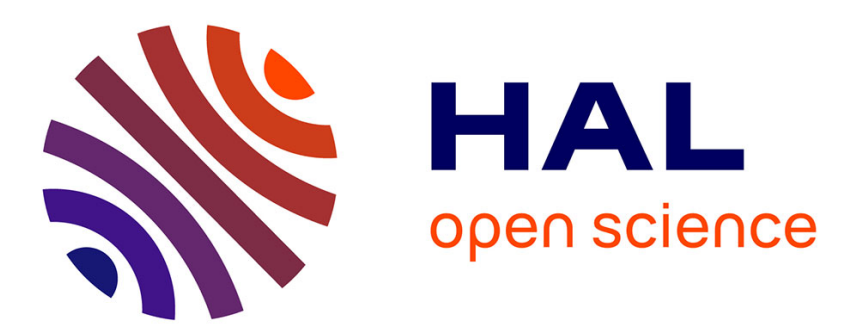

\title{
Convexity of injectivity domains on the ellipsoid of revolution: The oblate case
}

Bernard Bonnard, Jean-Baptiste Caillau, Ludovic Rifford

\section{To cite this version:}

Bernard Bonnard, Jean-Baptiste Caillau, Ludovic Rifford. Convexity of injectivity domains on the ellipsoid of revolution: The oblate case. Comptes Rendus. Mathématique, 2010, 348 (23-24), pp.13151318. 10.1016/j.crma.2010.10.036 . hal-01336325

\section{HAL Id: hal-01336325 \\ https://hal.science/hal-01336325}

Submitted on 23 Jun 2016

HAL is a multi-disciplinary open access archive for the deposit and dissemination of scientific research documents, whether they are published or not. The documents may come from teaching and research institutions in France or abroad, or from public or private research centers.
L'archive ouverte pluridisciplinaire HAL, est destinée au dépôt et à la diffusion de documents scientifiques de niveau recherche, publiés ou non, émanant des établissements d'enseignement et de recherche français ou étrangers, des laboratoires publics ou privés. 


\title{
Convexity of injectivity domains on the ellipsoid of revolution: The oblate case
}

\author{
Bernard Bonnard $^{\mathrm{a}}$, Jean-Baptiste Caillau ${ }^{\mathrm{a}}$ and Ludovic Rifford ${ }^{\mathrm{b}}$ \\ ${ }^{a}$ Institut math., Univ. Bourgogne \& CNRS, 9 avenue Savary, F-21078 Dijon \\ ${ }^{\mathrm{b}}$ Labo. Dieudonné, Univ. Nice \& CNRS, Parc Valrose, F-06108 Nice \\ Received *****; accepted after revision +++++ \\ Presented by
}

\begin{abstract}
We characterize the convexity properties of the tangent injectivity domain on an the ellipsoid of revolution in the oblate case. To cite this article: Bonnard, B.; Caillau, J.-B.; Rifford, L. C. R. Acad. Sci. Paris, Ser. I xxx (200x).

\section{Résumé}

Convexité des domaines d'injectivité sur l'ellipsoïde de révolution : le cas oblat. On caractérise les propriétés de convexité du domaine d'injectivité sur un ellipsoïde de révolution oblat. Pour citer cet article : Bonnard, B. ; Caillau, J.-B. ; Rifford, L. C. R. Acad. Sci. Paris, Ser. I xxx (200x).
\end{abstract}

\section{Introduction}

The purpose of the present note is to study convexity properties of injectivity domains on the oblate ellipsoid of revolution given in $\mathbf{R}^{3}$ by the cartesian equation

$$
E_{\mu}: \quad x^{2}+y^{2}+\left(\frac{z}{\mu}\right)^{2}=1,
$$

with unit semi-major axis and semi-minor axis of length $\mu \in(0,1]$. To this aim, we use the covering of $E_{\mu}$ minus its poles

$$
\mathbf{R} \times(0, \pi) \ni(\theta, \varphi) \longmapsto(\sin \varphi \cos \theta, \sin \varphi \sin \theta, \mu \cos \varphi),
$$

Email addresses: bernard.bonnardeu-bourgogne.fr(Bernard Bonnard), jean-baptiste.caillau@u-bourgogne.fr (Jean-Baptiste Caillau), ludovic.rifford@math.cnrs.fr(Ludovic Rifford). 
and consider the metric

$$
\mathrm{d} s^{2}=X \mathrm{~d} \theta^{2}+(1-X / \lambda) \mathrm{d} \varphi^{2}
$$

where $X=\sin ^{2} \varphi$ and $\lambda=1 /\left(1-\mu^{2}\right) \in(1, \infty]$. It can be put in polar form setting

$$
\mathrm{d} \psi=\mathrm{d} \varphi \sqrt{1-X / \lambda}
$$

which amounts to introducing the elliptic function of second kind $\psi=E(\varphi, k)$ with modulus $k^{2}=1 / \lambda$. According to standard Riemannian geometry [1], for any geodesic but equator and meridians, $\psi$ and $\varphi$ are periodic with the same period $T$. In consequence, a geodesic on the oblate ellipsoid of revolution is either a meridian circle, or the equator, or a curve symmetric with respect to the equator such that the $\psi$ component is periodic. Geodesics are integral curves of the Hamiltonian

$$
H=\frac{1}{2}\left(\frac{p_{\theta}^{2}}{X}+\frac{p_{\varphi}^{2}}{1-X / \lambda}\right)
$$

where $p=\left(p_{\theta}, p_{\varphi}\right)$ is the adjoint vector. The coordinate $\theta$ is cyclic, and $p_{\theta}=$ constant is the Clairaut relation. The Hamiltonian flow allows to define the exponential mapping on $\mathbf{R} \times H\left(x_{0}, .\right)^{-1}(\{1 / 2\})$ by

$$
\exp _{x_{0}}\left(t, p_{0}\right)=x\left(t, x_{0}, p_{0}\right) \text {. }
$$

As a subset of the cotangent bundle, the injectivity domain is defined as

$$
I\left(x_{0}\right)=\left\{t p_{0} \mid t \in\left[0, t_{\mathrm{cut}}\left(x_{0}, p_{0}\right)\right], H\left(x_{0}, p_{0}\right)=1 / 2\right\}
$$

where the cut time $t_{\text {cut }}\left(x_{0}, p_{0}\right)$ is the supremum of times $t>0$ such that the curve $\exp _{x_{0}}\left(\cdot, p_{0}\right)$ is minimizing from $x_{0}=\exp _{x_{0}}\left(0, p_{0}\right)$ to $\exp _{x_{0}}\left(t, p_{0}\right)$. Since $E_{\mu}$ is symmetric with respect to the equator with Gaussian curvature nondecreasing from the North pole to the equator, the cut time may be shown $[4,10]$ to satisfy (excluding the equator)

$$
t_{\text {cut }}\left(x_{0}, p_{0}\right)=t_{\text {cut }}\left(p_{\theta}\right)=\frac{T\left(p_{\theta}\right)}{2}
$$

where $T\left(p_{\theta}\right)$ denotes the period of the $\varphi$ variable.

Convexity properties are identical whether the domain is expressed on the tangent or on the cotangent bundle since the corresponding change of coordinates is the linear Legendre transform. The domain is convex if and only if its boundary is a convex curve, that is a curve with constant sign curvature. The aim of next section is to sketch a proof of the following.

Theorem 1.1 The injectivity domain on an oblate ellipsoid of revolution is convex for any point if and only if the ratio between the minor and the major axes is greater or equal to $1 / \sqrt{3}$.

It is worth noticing that the convexity issue plays a crucial role in the regularity theory of optimal transport maps with quadratic cost on Riemannian manifolds (see the monograph [12] for more on optimal transportation). In [9], the convexity of all injectivity domains together with the Ma-Trudinger-Wang condition, is shown to be necessary and sufficient for a compact Riemannian surface to satisfy the so-called transport continuity property (Theorem 1.3, loc. cit.). Therefore, Theorem 1.1 shows that any oblate ellipsoid of revolution with $\mu<1 / \sqrt{3}$ cannot satisfy this property. In [7], this was proven for $\mu \leq 0.29<1 / \sqrt{3}$ (Corollary 5.1, loc. cit.). According to [8, Theorem 1.1], convexity of all injectivity domains holds for any small enough $\mathscr{C}^{4}$ perturbation of the round metric on the sphere. As a consequence, the injectivity domains of any oblate ellipsoid of revolution close enough to the round sphere $(\mu=1)$ are always convex. Furthermore, it may be shown that convexity of all injectivity domains is lost in the singular Riemannian case $\mu=0$ (see the argument below) and a fortiori on oblate ellipsoids with small enough semi-minor axis. Theorem 1.1 confirms these facts and shows indeed that the only issue for convexity is the value of $\mu$ with respect to $1 / \sqrt{3}$. 
Besides its own geometric interest, the oblate ellipsoid case is also related to the optimal control of two bodies in space mechanics. It is shown in $[2, \S 3]$ that this control problem leads to study on the two-sphere a one-parameter family of metrics ${ }^{1}$ which are conformal to the canonical one on an oblate ellipsoid of revolution. This allows to interpretate the parameter as the ratio $\mu$ between the minor and major axes of the conformal ellipsoid. ${ }^{2}$

\section{Sketch of the proof}

Given $\lambda$ and $\varphi_{0}\left(\theta_{0}\right.$ can be set to zero thanks to the symmetry of revolution), the level set $H=1 / 2$ is parameterized according to

$$
p_{\theta}=\cos \alpha \sqrt{X_{0}}, \quad p_{\varphi}=\sin \alpha \sqrt{1-X_{0} / \lambda}, \quad \alpha \in[0,2 \pi],
$$

with $X_{0}=\sin ^{2} \varphi_{0}$. Because of symmetries, convexity has only to be checked on a quarter of the curve, $\alpha \in[0, \pi / 2]$. The boundary of the injectivity domain on the cotangent space is

$$
\alpha \mapsto \frac{T\left(p_{\theta}\right)}{2}\left(p_{\theta}, p_{\varphi}\right),
$$

so the curvature condition is expressed as a sign condition on the quantity

$$
T\left(T+p_{\theta} T^{\prime}\right)+\left(X_{0}-p_{\theta}^{2}\right)\left(2 T^{2}-T T^{\prime \prime}\right), \quad p_{\theta} \in\left[0, \sqrt{X_{0}}\right],
$$

where $^{\prime}=\mathrm{d} / \mathrm{d} p_{\theta}$ and where $T$ implicitly also depends on the parameter $\lambda$. The quadrature on $\varphi$ is parameterized by the algebraic complex curve

$$
\left[\frac{\dot{X}(\lambda-X)}{\sqrt{\lambda}}\right]^{2}=4\left(X-p_{\theta}^{2}\right)(X-1)(X-\lambda)
$$

which is of genus one excluding the following degeneracies. When $X_{0}=1\left(\varphi_{0}=\pi / 2\right), p_{\theta}=1$ defines the equator. The curve also degenerates to a rational surface for $\lambda=\infty(\mu=1$-round sphere $)$ or $\lambda=1$ ( $\mu=0$ - flat ellipsoid $)$. In the latter case, as the induced metric on the two-sided disk is flat (see $[3, \S 4])$, the injectivity domain for $\mu$ close to 0 and $\varphi_{0}$ close to $\pi / 2$ is by continuity a deformation of the union at the origin of two disjoint disks (eight-shaped domain), hence not convex. Conversely, for $\mu$ close to 1 , the metric is $\mathscr{C}^{4}$-close to the round one and convexity must hold for an arbitrary initial condition (see [6, Theorem 1.3] or [8, Theorem 1.1]).

Setting

$$
u=X-\frac{p_{\theta}^{2}+1+\lambda}{3} \text { and } v=\frac{\dot{X}(\lambda-X)}{\sqrt{\lambda}},
$$

we get the Weierstrass parameterization by $v^{2}=4 u^{3}-g_{2} u-g_{3}$ with invariants rational in the parameters,

$$
\begin{gathered}
g_{2}=\frac{4}{3}\left[p_{\theta}^{4}-(\lambda+1) p_{\theta}^{2}+\left(\lambda^{2}-\lambda+1\right)\right] \\
g_{3}=\frac{4}{27}\left[2 p_{\theta}^{6}-3(\lambda+1) p_{\theta}^{4}-3\left(\lambda^{2}-4 \lambda+1\right) p_{\theta}^{2}+\left(2 \lambda^{3}-3 \lambda^{2}-3 \lambda+2\right)\right] .
\end{gathered}
$$

Since $X$ is in $\left[p_{\theta}^{2}, 1\right], u$ belongs to $\left[e_{2}, e_{3}\right]$ where the three roots of the cubic are

$$
e_{1}=\frac{2 \lambda-p_{\theta}^{2}-1}{3}, \quad e_{2}=\frac{2 p_{\theta}^{2}-1-\lambda}{3}, \quad e_{3}=\frac{2-p_{\theta}^{2}-\lambda}{3} .
$$

1. Also independently introduced in [5]

2. Surprisingly, the Gauss curvature of these metrics is nondecreasing from the North the pole to the equator (which ensures a simple structure of cut loci [10, Main Theorem]) if and only if $\mu$ is again greater or equal to $1 / \sqrt{3}$. 
The parameterization uses the bounded component of the real cubic, that is $z \in \omega^{\prime}+\mathbf{R}$ where $\omega \mathbf{Z}+\omega^{\prime} \mathbf{Z}$ is the real rectangular lattice of periods of $u(z)=\wp(z)$. The time law is

$$
\frac{\mathrm{d} t}{\mathrm{~d} z}=\frac{\lambda-X}{\sqrt{\lambda}}
$$

so the period of $\varphi$, which is twice the period of $X$, equals

$$
T=\frac{4}{\sqrt{\lambda}}\left(e_{1} \omega+\eta\right), \quad \eta=\zeta(\omega) .
$$

Differentiating the period $\omega$ and the quasi-period $\eta$ with respect to the invariants $g_{2}, g_{3}$ (see [11, pp. 307-308]), the derivatives in $p_{\theta}$ are obtained as linear combinations in $\omega$ and $\eta$ with coefficients in $\mathbf{R}\left(\lambda, p_{\theta}\right)$. One has

$$
\partial \omega=-A \omega-B \eta, \quad \partial \eta=C \omega+A \eta,
$$

where

$$
\begin{gathered}
\partial=\delta \frac{\mathrm{d}}{\mathrm{d} p_{\theta}}, \quad \delta=\frac{3\left(p_{\theta}^{2}-1\right)\left(p_{\theta}^{2}-\lambda\right)}{p_{\theta}} \\
A=2 p_{\theta}^{2}-(\lambda+1), \quad B=3, \quad C=\frac{1}{3}\left[p_{\theta}^{4}-(\lambda+1) p_{\theta}^{2}+\left(\lambda^{2}-\lambda+1\right)\right] .
\end{gathered}
$$

Define $\tau=3 T \sqrt{\lambda} / 4$. The derivatives up to second order of $\tau$ are

$$
\begin{gathered}
\tau=-\left(p_{\theta}^{2}-2 \lambda+1\right) \omega+3 \eta, \quad \tau^{\prime}=\frac{p_{\theta}}{p_{\theta}^{2}-1}\left[-\left(p_{\theta}^{2}+\lambda-2\right) \omega+3 \eta\right], \\
\tau^{\prime \prime}=\frac{\left[(2 \lambda-1) p_{\theta}^{4}-\left(\lambda^{2}+1\right) p_{\theta}^{2}-\lambda(\lambda-2)\right] \omega+3\left[(\lambda-2) p_{\theta}^{2}+\lambda\right] \eta}{\left(p_{\theta}^{2}-1\right)^{2}\left(p_{\theta}^{2}-\lambda\right)} .
\end{gathered}
$$

We only provide a sketch of proof that involves the two following lemmas.

Lemma 2.1 For any $\lambda>1$ and $X_{0} \in[0,1)$, the (normalized) curvature

$$
\kappa\left(p_{\theta}, X_{0}, \lambda\right)=\tau\left(\tau+p_{\theta} \tau^{\prime}\right)+\left(X_{0}-p_{\theta}^{2}\right)\left(2 \tau^{\prime 2}-\tau \tau^{\prime \prime}\right)
$$

is decreasing in $p_{\theta}$ on $\left[0, \sqrt{X_{0}}\right]$.

The worst case is so obtained for $p_{\theta}=\sqrt{X_{0}}$, and the sign of $\kappa_{2}$ defined according to

$$
\kappa_{2}\left(X_{0}, \lambda\right)=\tau+\tau^{\prime} \sqrt{X_{0}}
$$

has to be checked for $X_{0} \in[0,1]$.

Lemma 2.2 For any $\lambda>1, \kappa_{2}$ is decreasing in $X_{0}$ on $[0,1)$.

The function $\kappa_{2}$ degenerates as $X_{0} \rightarrow 1$ (equator). Now,

$$
\kappa_{2}=\frac{\omega}{1-X_{0}}\left[(\lambda-1-3 \chi)+(\lambda-2+6 \chi)\left(1-X_{0}\right)+2\left(1-X_{0}\right)^{2}\right], \quad \chi=\frac{\eta}{\omega} .
$$

The degeneracy of $\chi$ is known [11, p. 314], and the previous differentiation rules imply

$$
\partial \chi=C+2 A \chi+B \chi^{2}
$$

which allows to obtain an asymptotic of first order of $\chi$ when $X_{0} \rightarrow 1$. Finally,

$$
\kappa_{2}=\frac{3 \pi}{2 \sqrt{\lambda-1}}\left(\lambda-\frac{3}{2}\right)+o(1), \quad X_{0} \rightarrow 1,
$$

hence the zero for $\lambda=3 / 2$, that is for $\mu=1 / \sqrt{3}$. As a result, domains of injectivity of the oblate ellipsoid are convex (for any initial condition) if and only if the semi-minor axis is not less than $1 / \sqrt{3}$. Below this limit, there are always initial conditions such that convexity is lost. 


\section{References}

[1] Besson, G. Géodésiques des surfaces de révolution. Séminaire de théorie spectrale et géométrie S9 (1991), 33-38.

[2] Bonnard, B; Caillau, J.-B. Optimality results in orbit transfer. C. R. Acad. Sci. Paris, Ser. I 345 (2007), 319-324.

[3] Bonnard, B; Caillau, J.-B. Singular metrics on the two-sphere in space mechanics. HAL preprint (2008), no. 00319299, 1-25.

[4] Bonnard, B.; Caillau, J.-B.; Sinclair, R.; Tanaka, M. Conjugate and cut loci of a two-sphere of revolution with application to optimal control. Ann. Inst. H. Poincaré Anal. Non Linéaire 26 (2009), no. 4, 1081-1098.

[5] Faridi, A. M.; Schucking, E. L. Geodesics and deformed spheres. Proc. Amer. Math. Soc. 100 (1987), no. 3, 522-525.

[6] Figalli, A.; Rifford, L. Continuity of optimal transport maps and convexity of injectivity domains on small deformations of $\mathbf{S}^{2}$. Comm Pure Appl. Math. 62 (2009), no. 12, 1670-1706.

[7] Figalli, A.; Rifford, L.; Villani, C. On the Ma-Trudinger-Wang curvature on surfaces. Calc. Var. Partial Differential Equations (to appear)

[8] Figalli, A.; Rifford, L.; Villani, C. Nearly round spheres look convex. Preprint, 2010.

[9] Figalli, A.; Rifford, L.; Villani, C. Necessary and sufficient conditions for continuity of optimak transport maps on Riemannian manifolds. Preprint, 2010.

[10] Sinclair, R.; Tanaka, M. The cut locus of a two-sphere of revolution and Topogonov's comparison theorem. Tohoku Math. J. 59 (2007), no. 2, 379-399.

[11] Halphen, G.-H. Traité des fonctions elliptiques et de leurs applications, Première Partie. Gauthier-Villars, 1886.

[12] Villani, C. Optimal transport, old and new. Vol. 338, Springer, 2009. 